\title{
A SEMI ANALYTICAL ITERATIVE TECHNIQUE FOR SOLVING DUFFING EQUATIONS
}

\author{
M.A. Al-Jawary ${ }^{1} \S$, S.G. Al-Razaq ${ }^{2}$ \\ ${ }^{1,2}$ College of Education for Pure Sciences \\ Ibn-AL-Haithem \\ Baghdad University \\ Baghdad, IRAQ
}

\begin{abstract}
This paper presents new implementation of reliable iterative method proposed by Temimi and Ansari namely (TAM) for analytic and numerical solutions of Duffing equations in two types: damping and undamping equations. The solution is obtained in the series form that converge to the exact solution with easily computed components. Several problems are solved to demonstrate the efficiency of the proposed methods without any restrictive assumptions for nonlinear terms. However, for some examples numerical calculations evidenced by tables and figures for the analysis of the maximal error remainder values. The software used for the calculations in this study was MATHEMATICA ${ }^{\circledR} 9.0$.
\end{abstract}

Key Words: Duffing equation, damping equation, undamping equation, exact solution, iterative method

\section{Introduction}

Nonlinear differential equations play important role in modeling numerous important phenomena occurring in various fields of physics, chemistry and engineering science are frequently modeled through nonlinear differential equations.

Received: May 1, 2016

Published: August 16, 2016

${ }^{\S}$ Correspondence author
(C) 2016 Academic Publications, Ltd. url: www.acadpubl.eu 
Various methods have been used to solve linear and nonlinear differential equations such as A domain decomposition method, variational iteration method and some other analytic and approximate methods.

In 1918, the German electrical engineer Georg Duffing finds out the Duffing equation [4]. Duffing equation is a nonlinear differential equation, which is playing an important role in many sciences such as physics and engineering [5].

This equation that a occur as a result of vibrations of a mass of objects to a nonlinear spring force and periodic forcing [6]. Moon and Holmes, they conducted a modification on the general Duffing equation on the linear part that becames negative [7].

Duffing equation, it has become a basic role in many areas like a classical oscillator is chaotic systems [8], nonlinear vibration of beams and plates, research large amplitude oscillation of centrifugal governor systems [9], [10], periodic orbit extraction, nonlinear mechanical oscillators and prediction of diseases [8], [11], magnetic- pliancy mechanical systems [10], the nonlinear dynamics of slender elastica and the generalized pochhammer-chree (PC) equation [5].

This equation, it resolves in many of a new process in the open literature such as modified differential transform method [10], variational iteration method [12], Daftardar-Jafari (DJ) method [7], homotopy perturbation transform method [13], an effective approach method [11], a new approach method [14] and homotopy analysis method [15]. Several of this method are used to find analytic and numerical solution.

The Adomain decomposition method in [16] is successfully applied to solve Duffing equations, however, it needs to calculate Adomain polynomial to deal with the nonlinear terms.

In this article, the general form of Duffing equation [5], will be solved:

$$
u^{\prime \prime}(x)+k_{1} u^{\prime}(x)+k_{2} u(x)+k_{3} u^{3}(x)=f(x)
$$

with initial condition

$$
u(0)=a, u^{\prime}(0)=b .
$$

where $k_{1}, k_{2}, k_{3}, a$ and $b$ are real constants. Duffing equation clearly is a nonlinear ordinary differential equation and it is of the second order [4].

The main aim of this paper is to implement an iterative method namely (TAM) which proposed by Temimi and Ansari in 2011 [1] to solve Duffing equations in two kind, damping and undamping. This method has successfully solved the nonlinear second order multipoint boundary value problems [2], nonlinear ordinary differential equations and system of nonlinear differential equations [3]. 
The present article has been arranged as follows, in Section 2, the basic idea of TAM is presented. In Section 3, the convergence and error analysis of the TAM are given. In Section 4, solving the Duffing equation by the proposed method will be illustrated. In Section 5, some test example of Duffing equation: damping and undamping will be solved and finally in Section 6 the conclusion is presented.

\section{The Basic Idea of TAM}

We rewrite the Eq.(1) as

$$
L(u(x))+N(u(x))+g(x)=0,
$$

with boundary condition

$$
B\left(u, u^{\prime}\right)
$$

where $x$ indicates the independent variable, $u(x)$ ia an unknown function, $g(x)$ is a known function, $L$ is a linear operator, $N$ is a nonlinear operator. Here we can take view linear parts and add them to $N$ as needed. The method applied as follows, we start by suppose that $u_{0}(x)$ is an initial valuation of the solution to the equation $u(x)$ and is the solution of the equation

$$
L\left(u_{0}(x)+g(x)=0, \quad \text { with } \quad B\left(u_{0}, u_{0}^{\prime}\right) .\right.
$$

To find the next iterate to the solution, we solve the following equation:

$$
L\left(u_{1}+g(x)+N\left(u_{0}(x)=0, \quad \text { with } \quad B\left(u_{1}, u_{1}^{\prime}\right) .\right.\right.
$$

Thus, we have a simple iterative step which is effecting the solution of a linear set of equation i. e.,

$$
L\left(u_{n+1}(x)\right)+g(x)+N\left(u_{n}(x)\right)=0, \quad \text { with } \quad B\left(u_{n+1}, u_{n+1}^{\prime}\right) .
$$

It is noted that each of the $u_{i}(x)$ are solutions to Eq.(3). We suggest that the convergence of the iteration be monitored using standard error control procedures such as the maximal error reminder as illustrated in [3]. 


\section{Convergence and Error Analysis of the TAM}

Consider the $L^{2}$ norm [3]

$$
\|f\|=\left(\int_{0}^{t} f^{2}\right)^{\frac{1}{2}} .
$$

The error remainder is given by [7]

$$
E R_{n}=u_{n}^{\prime \prime}(x)+k_{1} u_{n}^{\prime}(x)+k_{2} u_{n}(x)+k_{3} u_{n}^{3}(x)-f(x) .
$$

The maximal error remainder parameters are :

$$
M E R_{n}=\max _{0 \leq x \leq 1}\left|E R_{n}(x)\right| .
$$

Consider the Duffing equation

$$
u^{\prime \prime}(x)+k_{1} u^{\prime}(x)+k_{2} u(x)+k_{3} u^{3}(x)-f(x)=0,
$$

with initial condition

$$
u(0)=a \quad \text { and } \quad u^{\prime}(0)=b
$$

Follows we prove the sequence of function $u_{n}$, which are solutions of [3]

$$
u_{n+1}^{\prime \prime}(x)=-k_{1} u_{n}^{\prime}(x)-k_{2} u_{n}(x)-k_{3} u_{n}^{3}(x)+f(x),
$$

with initial condition

$$
u_{n}(0)=a \quad \text { and } \quad u_{n}^{\prime}(0)=b .
$$

Converges to the solution of Eqs.(11)-(12), where $f$ is a nonlinear analytic function and the initial guess function $u_{0}$ can be taken as a solution of the initial problem

$$
L\left(u_{0}(x)+f(x)=0,\right.
$$

with initial condition

$$
u_{0}(0)=a, \quad u_{0}^{\prime}(0)=b .
$$

If we study the convergence of the above iterative method, we need to state the Green's function [17], associated with Eq.(11), (12) that is

$$
G(x, t)=A x+B \quad \text { for } \quad x<t \quad \text { and } \quad G(x, t)=C x+D \quad \text { for } \quad x>t \text {, }
$$


We apply the condition

$$
\varliminf_{x \rightarrow \infty} G(x, t)=\varliminf_{x \rightarrow \infty} \frac{d G(x, t)}{d x}=0 .
$$

If $C=D=0$ the continuity and jump conditions at $x=t$ then yield

$$
G(x, t)=t-x \quad \text { for } \quad x<t \quad \text { and } \quad G(x, t)=0 \quad \text { and } \quad x>t .
$$

Let

$$
K=\max _{x, t}|G(x, t)|=\max _{x, t}|t-x| .
$$

We put this Green's function in coming formula [17]

$$
u=\int_{0}^{\infty} G(x, t) f(x) d x-p(0)\left[a \frac{d G(x, t)}{d x}-G(0, t) b\right] .
$$

If $p=1$, we have

$$
u=\int_{0}^{\infty} G(x, t)\left(u^{\prime \prime}(t)+k_{1} u^{\prime}(t)+k_{2} u(t)+k_{3} u^{3}(t)-f(t)\right) d t+a+b t .
$$

The sequence of solution of Eq.(13), (14) as:

$$
u_{n+1}=\int_{0}^{t} G(x, t)\left(u_{n}^{\prime \prime}(t)+k_{1} u_{n}^{\prime}(t)+k_{2} u_{n}(t)+k_{3} u_{n}^{3}(t)-f(t)\right) d t .
$$

Be subtracting Eq.(22), (23) and applying the Mean value theorem, we have

$$
u_{n+1}-u=\int_{0}^{t} G(x, t) f_{u}\left(\theta_{n}\right)\left(u_{n}(t)-u(t)\right) d t
$$

where

$$
\theta_{n} \epsilon\left(u_{n}, u\right) \text { and } \quad f_{u}=\frac{d f}{d u}
$$

Let

$$
M=\max _{u_{\mathrm{n}}<\theta_{\mathrm{n}}<u}\left|f_{u}\left(\theta_{n}\right)\right|
$$

Theorem 3.1.1. (see [3]) Let $u$ and $u_{n}$, respectively, be the solutions of Eq.(11), (12) and Eq.(13), (14). Suppose that $f$ is a nonlinear analytic function. Then, if $M k b<1$, the sequence of functions $u_{n}$ converges to the exact solution $u$ in the $L^{2}$ - norm, where $M$ and $K$ are defined, respectively, by Eq.(26) and (20). 
Theorem 3.1.2. (see [3]) Let $u$ and $u_{n}$, respectively, be the solutions of Eq.(11), (12) and Eq.(13), (14). Suppose that $f$ is a nonlinear analytic function. Then, if $M k b<1$, the maximal error remainder defined by (6) converges to zero with respect to $n$ and therefore the sequence of functions converges to the exact solution $u$, where $M$ and $K$ are defined by Eq.(26) and (20), respectively.

\section{Solving Duffing Equations by using the TAM}

Here in this section, we apply the TAM to obtain the exact and approximate solution for Duffing equations. Consider a form of the Duffing equation given in Eq.(1).

We write the Duffing equation as operator form as follows:

$$
L(u(x))+N(u(x))+g(x)=0,
$$

where

$$
N(u(x))=k_{1} u^{\prime}(x)+k_{2} u(x)+k_{3} u^{3}(x), \text { and } \quad g(x)=-f(x),
$$

with initial conditions

$$
u(0)=a \quad, u^{\prime}(0)=b
$$

where $x$ denotes the independent variable, $u(x)$ is an unknown function, $g(x)$ is a known function, $L$ is a linear operator and $N$ is a nonlinear operator. Certainly the main requirement here is that $L$ is the linear part of the Duffing equation. The method we apply works in the following way, we start by assuming that $u_{0}(X)$ is an initial guess of the solution to the problem $u(X)$ and is the solution of the equation

$$
L\left(u_{0}(x)\right)+g(x)=0, \text { with } \quad u_{0}(0)=a \quad \text { and } \quad u_{0}^{\prime}(0)=b
$$

The next iteration, we solve the following equation:

$$
L\left(u_{1}(x)\right)+g(x)+N\left(u_{0}(x)\right)=0, \text { with } \quad u_{1}(0)=a \quad \text { and } \quad u_{1}^{\prime}(0)=b .
$$

Thus we have a simple iterative restraint which is actively the solution of a linear set of equation i.e.,

$$
L\left(u_{n+1}(x)\right)+g(x)+N\left(u_{n}(x)\right)=0, \text { with } u_{n+1}(0)=a \text { and } u_{n+1}^{\prime}(0)=b .
$$

It is interesting to point out here, each of the $u_{i}(\mathrm{x})$ is a solution to Eq. (27). 


\section{Test Examples}

In this section, we will evaluate the efficiency of the TAM through applying this method for some examples of the damping and undamping Duffing equations. Some of these examples have analytic solution and others have numerical solution. Also, we calculate the error remainder with the maximal error remainder parameters in Eqs.(9) and (10) for the approximate solution.

\subsection{Damping Duffing Equation}

Example 1. Let us consider the damping Duffing equation [18]:

$$
u^{\prime \prime}+2 u^{\prime}+u+8=1-3 x
$$

with initial condition:

$$
u(0)=\frac{1}{2}, \quad \text { and } \quad u^{\prime}(0)=-\frac{1}{2}
$$

Now, we apply the TAM by first distributing the equation as:

$$
L(u)=u^{\prime \prime}, N(u)=2 u^{\prime}+u+8 \text { and } g(x)=1-3 x,
$$

Thus the initial problem it

$$
L\left(u_{0}\right)=1-3 x, \text { with } \quad u_{0}(0)=\frac{1}{2} \quad \text { and } \quad u_{0}^{\prime}=-\frac{1}{2}
$$

We subsequent problems can be obtained from the iterative problem generating relation

$$
L\left(u_{n+1}(x)\right)+g(x)+N\left(u_{n}(x)\right)=0, u_{n+1}(0)=\frac{1}{2}, \text { and } u_{n+1}^{\prime}(0)=-\frac{1}{2}
$$

Solving the initial problem as follows

$$
u_{0}^{\prime \prime}(x)=1-3 x
$$

By integrating both sides of equation (37) from 0 to $x$, we obtain

$$
\int_{0}^{x} u_{0}^{\prime \prime}(t) d t=\int_{0}^{x}(1-3 t) d t
$$

Then after applying the condition $u_{0}^{\prime}(0)=-\frac{1}{2}$ in Eq. (38), we get 


$$
u_{0}^{\prime}=-\frac{1}{2}+x-\frac{3 x^{2}}{2},
$$

Integrate Eq.(39) again from 0 to $x$, we obtain

$$
\int_{0}^{x} u_{0}^{\prime}(t) d t=\int_{0}^{x}\left(-\frac{1}{2}+t-\frac{3 t^{2}}{2}\right) d t
$$

Then, by applying the initial condition

$$
u_{0}(x)=\frac{1}{2}-\frac{x}{2}+\frac{x^{2}}{2}-\frac{x^{3}}{2},
$$

In a similar manner other iterations can be achieved.

The first iteration will be then

$$
u_{1}^{\prime \prime}=1-3 x-2 u_{0}^{\prime}-u_{0}-8 u_{0}^{3} \text { with } u_{1}(0)=\frac{1}{2} \text { and } u_{1}^{\prime}(0)=-\frac{1}{2}
$$

Then the solution of Eq.(42)

$$
u_{1}(x)=\frac{1}{2}-\frac{x}{2}+\frac{x^{2}}{4}-\frac{x^{3}}{4}-\frac{7 x^{4}}{24}+\frac{21 x^{5}}{40}-\ldots,
$$

The second iteration is

$$
u_{2}^{\prime \prime}=1-3 x-2 u_{1}^{\prime}-u_{1}-8 u_{1}^{3}, \quad \text { with } \quad u_{2}(0)=\frac{1}{2} \quad \text { and } \quad u_{2}^{\prime}(0)=-\frac{1}{2} \text {, }
$$

Then we have

$$
u_{2}(x)=\frac{1}{2}-\frac{x}{2}+\frac{x^{2}}{4}-\ldots,
$$

and so on. The approximate solution will be then:

$$
u(x)=\frac{1}{2}-\frac{x}{2}+\frac{x^{2}}{4}-\ldots,
$$

This has the closed form:

$$
u(x)=\frac{1}{2}\left(e^{-x}\right)
$$

Which the exact solution of Eq. (32) [18].

Example 2. We next consider the damping Duffing equation [14] 


$$
u^{\prime \prime}+u^{\prime}+u^{3}=0
$$

with initial condition

$$
u(0)=1 \quad \text { and } \quad u^{\prime}(0)=1
$$

We apply the TAM as follows

$$
L(u)=u^{\prime \prime} ; N(u)=u^{\prime}+u^{3} \quad \text { and } \quad g(x)=0
$$

This the initial problem is

$$
L\left(u_{0}\right)=0 \quad \text { with } \quad u_{0}(0)=1 \quad \text { and } \quad u_{0}^{\prime}(0)=1,
$$

Following problem can be obtained from the iterative problem generating relation

$$
L\left(u_{n+1}(x)\right)+g(x)+N\left(u_{n}(x)\right)=0 \text { with } u_{n+1}(0)=1 \text { and } u_{n+1}^{\prime}(0)=1,
$$

Solving the initial problem we have

$$
u_{0}=1+x,
$$

The first iteration can be carried through and is given as

$$
u_{1}^{\prime \prime}=-u_{0}^{\prime}-u_{0}^{3} ; \quad \text { with } \quad u_{1}(0)=1 \quad \text { and } \quad u_{1}^{\prime}(0)=1,
$$

Then has a solution

$$
u_{1}(x)=1+x-x^{2}-\frac{x^{3}}{2}-\frac{x^{4}}{4}-\frac{x^{5}}{20},
$$

Applying the same process for $u_{2}$ as follows

$$
u_{2}^{\prime \prime}=-u_{1}^{\prime}-u_{1}^{3}, \quad \text { with } \quad u_{2}(0)=1 \quad \text { and } \quad u_{2}^{\prime}(0)=1,
$$

We solve this problem, then we have

$$
u_{2}(x)=1+x-x^{2}-\frac{x^{3}}{6}+\frac{x^{4}}{8}+\frac{3 x^{5}}{8}+\frac{2 x^{6}}{15}-\ldots
$$

and so on. The solution is in a series form is given by:

$$
u(x)=1+x-x^{2}-\frac{x^{3}}{6}+\frac{x^{4}}{8}+\frac{3 x^{5}}{8}+\frac{2 x^{6}}{15}-\ldots
$$




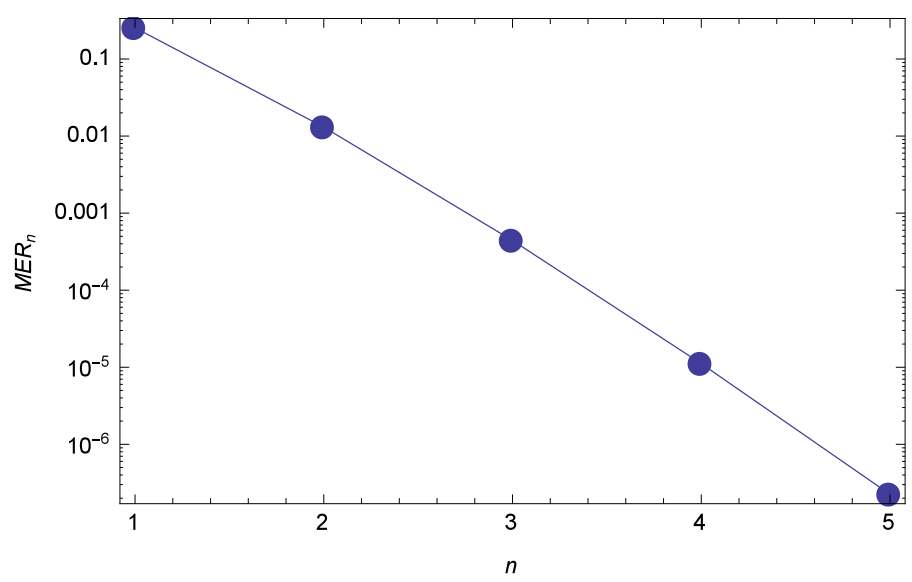

Figure 1: Logarithmic plots of MER versus $n$ is 1 through 5

The approximate solution of Eq. (48) can be further examined by evaluating the maximal error remainder in Table (1). It can be clearly seen that from Figure 1 the points are ley on a straight lines which mean exponential rate of convergence is achieved.

Table 1: the maximal error remainder for Eq. (21) using (TAM) where $n=$ $1, \ldots, 5$.

\begin{tabular}{|l|l|}
\hline $\mathrm{n}$ & MER \\
\hline 1 & 0.253868 \\
\hline 2 & 0.0130565 \\
\hline 3 & 0.000441662 \\
\hline 4 & 0.0000111419 \\
\hline 5 & $2.242097710 \times 10^{-7}$ \\
\hline
\end{tabular}

\subsection{Undamping Duffing Equation}

Example 3. Consider undamping Duffing equation [14]:

$$
u^{\prime \prime}+3 u-2 u^{3}=\cos (x) \sin (2 x)
$$

with initial condition

$$
u(0)=0 \quad \text { and } \quad u^{\prime}(0)=1
$$

We apply the proposed method by first distributing the equation as

$$
L(u)=u^{\prime \prime} ; N(u)=3 u-2 u^{3}, \text { and } g(x)=\text { series of }(\cos (x) \sin (2 x))
$$


Thus the initial problem is

$$
L\left(u_{0}\right)=2 x-\frac{7 x^{3}}{3}+\frac{61 x^{5}}{60}-\frac{547 x^{7}}{25920}+\ldots, \quad \text { with } \quad u_{0}(0)=0 \quad u_{0}^{\prime}(0)=1,
$$

We subsequent problems can be obtained from the analysis of this method

$$
L\left(u_{n+1}(x)\right)+g(x)+N\left(u_{n}(x)\right)=0, \text { with } u_{n+1}(0)=0 \text { and } u_{n+1}^{\prime}(0)=1,
$$

Solving the initial problem, then we have

$$
u_{0}(x)=x+\frac{x^{3}}{3}-\frac{7 x^{5}}{60}+\frac{61 x^{7}}{2520}-\frac{547 x^{9}}{181440}+\frac{703 x^{11}}{2851200}-\ldots,
$$

The first iteration will be

$$
u_{1}^{\prime \prime}=2 x-\frac{7 x^{3}}{3}+\frac{61 x^{5}}{60}-\frac{547 x^{7}}{25920}+\ldots-3 u_{0}+2 u_{0}^{3}, \text { with } u_{1}(0)=0 u_{1}^{\prime}(0)=1,
$$

Then the solution is

$$
u_{1}(x)=x-\frac{x^{3}}{3}-\frac{x^{5}}{15}+\frac{101 x^{7}}{1260}-\frac{407 x^{9}}{90720}-\ldots
$$

Applying the same process for $u_{2}$ as follows

$$
u_{2}^{\prime \prime}=2 x-\frac{7 x^{3}}{3}+\frac{61 x^{5}}{60}-\frac{547 x^{7}}{25920}+\ldots-3 u_{1}+2 u_{1}^{3}, \text { with } u_{2}(0)=0, u_{2}^{\prime}(0)=1,
$$

After solving, we have then

$$
u_{2}(x)=x-\frac{x^{3}}{6}+\frac{x^{5}}{120}-\ldots,
$$

and so on. The solution is in a series form is given in Taylor series form

$$
u(x)=\sin (x)
$$

Which the exact solution for Eq. (59) [14] obtained upon using the Taylor expansion for $\sin x$.

Example 4. Consider undamping Duffing equation [16]:

$$
u^{\prime \prime}+u+\varepsilon u^{3}=0
$$


with initial condition

$$
u(0)=1 \quad \text { and } \quad u^{\prime}(0)=5,
$$

We take the case when $\varepsilon=1$ then we have

$$
u^{\prime \prime}+u+u^{3}=0
$$

We apply the TAM as earlier by first distributing the equation as

$$
L(u)=u^{\prime \prime}, N(u)=u+u^{3} \quad \text { and } \quad g(x)=0,
$$

Thus the initial problem is

$$
L\left(u_{0}\right)=0 \quad \text { with } \quad u_{0}(0)=1 \quad \text { and } \quad u_{0}^{\prime}(0)=5,
$$

We subsequent problems can be obtained from the iteration of TAM then we get

$$
L\left(u_{n+1}(x)\right)+N\left(u_{n}(x)\right)=0, \quad \text { with } \quad u_{n+1}(0)=1 \quad \text { and } \quad u_{n+1}^{\prime}(0)=5,
$$

Solving the initial problem, we have

$$
u_{0}(x)=1+5 x
$$

The first iteration can be carried through and is given as

$$
u_{1}^{\prime \prime}=-u_{0}-u_{0}^{3}, \quad \text { with } \quad u_{1}(0)=1 \quad \text { and } \quad u_{1}^{\prime}(0)=5,
$$

Then, the solution will be

$$
u_{1}(x)=1+5 x-x^{2}-\frac{10 x^{3}}{3}-\frac{75 x^{4}}{12}-\frac{75 x^{5}}{12},
$$

Applying the same process for $u_{2}$ as follows

$$
u_{2}^{\prime \prime}=-u_{1}-u_{1}^{3}, \quad \text { with } \quad u_{2}(0)=1 \quad \text { and } \quad u_{2}^{\prime}(0)=5,
$$

This has a solution

$$
u_{2}(x)=1+5 x-x^{2}-\frac{10 x^{3}}{3}-\frac{71 x^{4}}{12}-\frac{49 x^{5}}{12}+\ldots,
$$

and so on. The solution is in a series form is given by: 


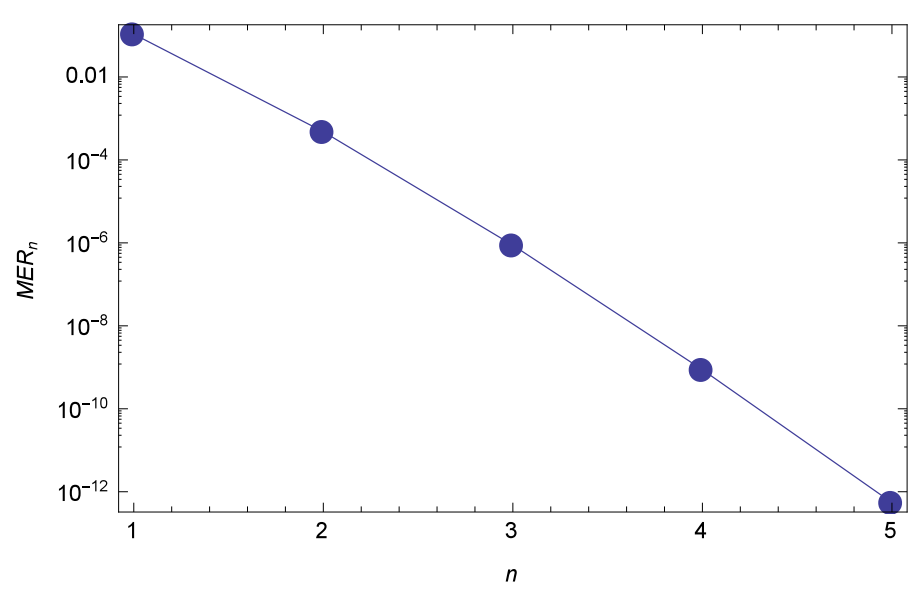

Figure 2: Logarithmic plots of MER versus $n$ is 1 through 5

$$
u(x)=1+5 x-x^{2}-\frac{10 x^{3}}{3}-\frac{71 x^{4}}{12}-\frac{49 x^{5}}{12}+\frac{587 x^{6}}{90} \ldots,
$$

The obtained series solution in Eq.(81) can be used for numerical solutions. The more components that we determine the highest accurncy level that we can achieve. This can be clearly seen in Table 2 and Figure 2.

Table 2: The maximal error remainder for Eq. (72) using TAM. where $n=1$, .., 5 .

\begin{tabular}{|l|l|}
\hline $\mathrm{n}$ & MER \\
\hline 1 & 0.10778 \\
\hline 2 & 0.000477836 \\
\hline 3 & $8.78127 \times 10^{-7}$ \\
\hline 4 & $8.75018 \times 10^{-10}$ \\
\hline 5 & $5.46 \times 10^{-13}$ \\
\hline
\end{tabular}

\section{Conclusion}

In this paper, we present an iterative method proposed by Tamimi and Ansari namely (TAM) for solving Duffing equation in two kind damping and undamping. This method is characterized by simplicity in applying without any restrictive assumptions for nonlinear terms. It is seems that the TAM appears to be very accurate to employ with reliable results and high accuracy. 


\section{References}

[1] H. Temimi, A. Ansari, A semi analytical iterative technique for solving nonlinear problems, Journal of Computers and Mathematics with Applications, 61 (2011) 203210, doi:10.1016/j.camwa.2010.10.042.

[2] H. Temimi, A. Ansari, A new iterative technique for solving nonlinear second order multi-point boundary value problems, Journal of Computers and Mathematics with Applications, 218 (2011) 1457- 1466, doi:10.1016/j.amc.2011.06.029.

[3] H. Temimi, A. Ansari, A computational iterative method for solving nonlinear ordinary differential equations, LMS J. Comput. Math, 18 (1) (2015) 730- 753, doi:10.1112/S1461157015000285.

[4] I. Kovacic, M. Brennan, The Duffing equation Nonlinear Oscillators and their Behaviour, John Wiley \& Sons Ltd, India 2011, Pag. 1. (Book), doi: 10.1002/9780470977859.

[5] A. Elias-Zuniga, Exact solution of the cubic- quantic Duffing oscillator, Journal of Applied Mathematical Modelling, 37 (2013) 2574- 2579, doi:10.1016/j.apm.2012.04.005.

[6] E. Agadjanov, Numerical solution of Duffing equation by the Laplace decomposition algorithm, Applied Mathematics and Computation 177 (2006) 572-580, doi:10.1016/j.amc.2005.07.072.

[7] M. Al- Jawary, S. Abd- Al- Razaq, Analytic and numerical solution for Duffing equations, International Journal of Basic and Applied Sciences, 5 (2) (2016) 115-119, doi: $10.14419 /$ ijbas.v5i2.5838.

[8] S. Balaji, A New Approach For Solving Duffing Equations Involving Both Integral And Non- Integral Forcing Terms, Ain Shams Engineering Journal, 5 (2014), 985-990, doi:10.1016/j.asej.2014.04.001.

[9] Z. Feng, G. Chen, S. Hsu, A qualitative Study of Damped Equation and Applications, Discrete and Continuous Dynamical Systems -Series B, Edinburg, 6(2005) 1097-1112, doi:10.3934/dcdsb.2006.6.1097.

[10] S. Nourazar, A. Mirzabeigy, Approximate Solution For Nonlinear Duffing Oscillator With Damping Effect Using The modified Differential Transform Method, Scientia Iranica, Transactions B: Mechanical Engineering 20 (2013) 364-368, doi:10.1016/j.scient.2013.02.023.

[11] M. Turkyilmazoglu, An Effective Approach For Approximate Analytical Solutions Of The Damped Duffing Equation, Physica Scripta, 86 (2012), 015301, doi:: 10.1088/0031-8949/86/01/015301.

[12] M. Khalid, M. Sultana, U. Arshad, M. Shoaib, A Comparison Between New Iterative Solutions of Non- Linear Oscillator Equation, International Journal of Computer Applications, 128(2015), 1-5, doi:10.5120/ijca2015906501.

[13] Y. Khan, H. Vazquez - Leal, N. Faraz, An Effective New Iterative Method For Oscillator Differential Equation, Scientia Iranica A, 19(2012), 1473-1477, doi:10.1016/j.scient.2012.10.018.

[14] B. Bulbul, M. Sezer, Numerical Solution of Duffing Equation by Using an Improved Taylor Matrix Method, Journal of Applied Mathematics, Volume 2013, Article ID 691614, 6 pages, http://dx.doi.org/10.1155/2013/691614. 
[15] L. Sheu, H. Chen, J. Chen, L. Tam, Chaotic Dynamics of the Fractionally Damped Duffing Equation, Chaos Solitons and Fractals, 32( 2007) 1459-1468, doi:10.1016/j.chaos.2005.11.066.

[16] M. Najafi, M. Moghimi, H. Massah, H. Khoramishad, M. Daemi, On the Application of Adomian Decomposition Method and Oscillation Equations, The 9th International Conference on Applied Mathematics, Istanbul, Turkey, 2006, doi=10.1.1.148.5625.

[17] A. Jerri, Introduction to Integral Equation with Applications, Marcel Dekker, 1985, doi: $10.1137 / 1028124$.

[18] G. Adomain, Solving frontier problems of physics: the decomposition method, Springerscience+ Business madia, USA (1994) 238-239 (Book),doi:10.1007/978-94-015-8289-6. 
